\title{
GPS-less Localization Protocol for Underwater Acoustic Networks
}

\author{
A.K Othman \\ Department of Electronic Engineering, Faculty of Engineering, \\ Universiti Malaysia Sarawak (UNIMAS) \\ Kota Samarahan, Sarawak, Malaysia. \\ Email: okhalid@feng.unimas.my
}

\begin{abstract}
The problem of underwater positioning is increasingly crucial due to the emerging importance of sub-sea activities. Knowledge of node location is essential for many applications for which sensor networks can be used. At the surface, positioning problems have been resolved by the extended use of GPS, which is straightforward and effective. Unfortunately, using GPS in the sub-sea environment is impossible and positioning requires the use of special systems. One of the major challenges in the underwater acoustic networks (UANs) area of research is the development of a networking protocol that can cope with the management of a dynamic sub-sea network. We propose a scheme to perform node discovery, using only one seed node (primary seed) in a known position. The discovery protocol can be divided into two parts: First, building up the relative co-ordinate system. Second, involving more remote nodes becoming seed nodes for further discoveries. Four different algorithms have been investigated; (i) Farthest/Farthest Algorithm, (ii) Farthest/Nearest Algorithm, (iii) Nearest/Farthest Algorithm and (iv) Nearest/Nearest Algorithm. We investigated the performances of random network topologies. Different locations of primary seed node were exercised and statistics for node discovery will be reported.
\end{abstract}

\section{INTRODUCTION}

Underwater acoustic networks can be formed by acoustically connected anchored nodes, autonomous underwater vehicles (AUVs), and it is possible to have a surface link that serves as a gateway to provide a communication link to an onshore station. Fig. 1 shows a generic underwater acoustic network.

An underwater network has several limitations compared to radio networks, most importantly the propagation delays which are very long with limited bandwidth. Another restriction that needs to be considered in UANs is the incapability of modems to transmit and receive signals at the same time (the near-far effect). To prevent the near-far effect which causes loss of data, scheduled transmission is required. The technique of node discovery must minimize the exchange of data in order to keep network management overheads to a minimum. Furthermore, in underwater acoustic networks, node connectivity is unpredictable. This connectivity depends upon several factors such as relative node orientation, noise level, propagation losses and fading. The connectivity is further affected by relative movement of the nodes, node and link failures and the addition of new nodes. Consequently, a very important characteristic of an underwater communication network is the ability to deal with changing topology.

To achieve full network functionality, nodes need to selforganize in an autonomous network which can adapt to the characteristics of the ocean environment. This paper addresses

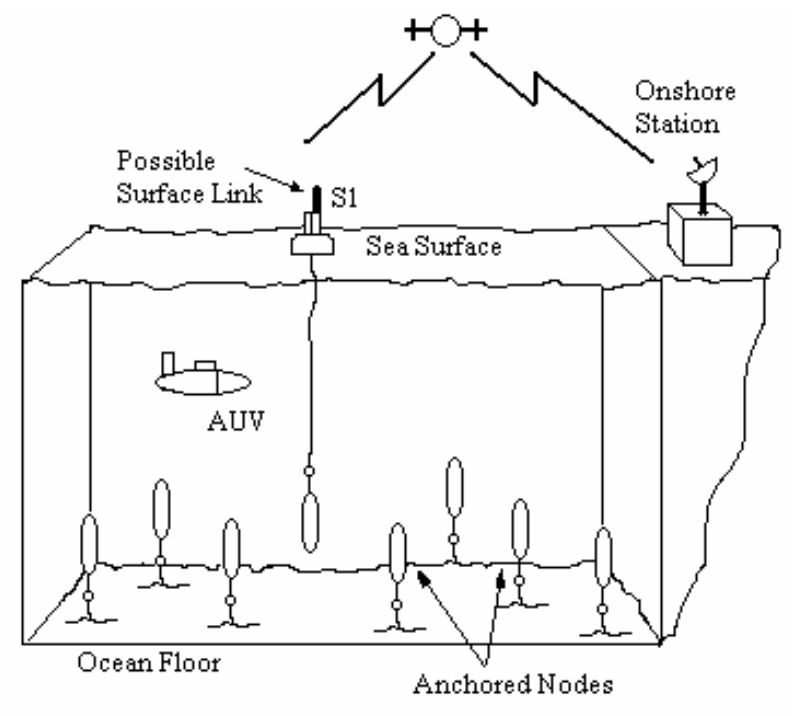

Fig. 1. Underwater acoustic network

the following problem: Given a set of nodes with unknown position co-ordinates, determine the relative co-ordinates of nodes.

\section{RELATED WORK}

In a localization system, several capabilities are necessary. First, the measurement techniques used to gain the information such as distance and other information. Second, the network discovery protocol which concerns the communication between nodes. Finally, techniques of deployment either using the anchor or beacon (nodes with known co-ordinate) or anchorfree bases.

The most popular measurement type is ranging. There are two methods used to obtain range measurements; timing and signal strength. Ranging is usually provided by estimating the distance to a neighbour by measuring the received signal strength (RSS) [1-3] from that neighbour, by time of arrival (ToA) [4] or by time difference of arrival (TDoA) [5].

In the ToA approach, the distance between a remote node and the beacon is measured by finding the one way propagation time between that node and the beacon. Geometrically, this provides a circle, centred on the beacon, on which the remote node must lie. By using at least three beacons to resolve ambiguities, the remote node's position is given by the intersection of the circles. In the TDoA approach, the time 